\title{
A test of alternative models of sentence recognition
}

\author{
SHERMAN W. TYLER and HENRY C. ELLIS \\ University of New Mexico, Albuquerque, New Mexico 87131
}

\begin{abstract}
A well-established finding in the sentence recognition paradigm of Bransford and Franks has been that recognition ratings increase as the number of ideas in the test sentences increases, with no difference in ratings for old and new sentences. Two major explanations of this phenomenon have been proposed. One, offered by Bransford and Franks, assumes subjects combine ideas in related sentences into single conceptual schemas and store little information about specific sentences. A second, set forth by Reitman and Bower, allows for more specific memory based on familiarity tags attached to ordered units and subunits of each sentence, such tags then being used as the basis of responding to test sentences. The relative plausibility of these two positions was evaluated by varying the precise nature of sentences seen in acquisition and recognition. Results generally favored the Bransford and Franks theory, but an unanticipated outcome suggested a possible modification of that theory.
\end{abstract}

The Bransford and Franks (1971) procedure has been frequently used in recent memory research. In this procedure, subjects are shown a number of sentences during an incidental learning task. Such sentences are constructed by combining one, two, or three of the simple ideas contained in four different four-idea reference sentences. After a group of these sentences has been shown, subjects are given an unexpected recognition test, during which they provide both an "old" or "new" response and a confidence rating for each sentence. In general, for test sentences containing only ideas from one reference sentence, recognition rating was found to be a linear function of the number of ideas in the test sentence, with no difference in confidence ratings for old and new sentences. In addition, four-idea sentences, which were always "new," were most highly recognized of all. On the other hand, test sentences combining ideas from two different reference sentences (noncases) received uniformly low confidence ratings. The explanation offered for these results is that, during acquisition, rather than storing each specific sentence as a separate memory trace, subjects construct individual schemata or holistic conceptual units for each idea set. Then, in recognition, judgments are based mainly on the number of ideas in a test sentence in common with the appropriate schema.

While this theoretical viewpoint has had an important impact, it has not been without its detractors. For example, Reitman and Bower (1973), have argued that the very nature of the Bransford and Franks (1971) paradigm introduces such memory overload and massive interference that it biases against finding any evidence

This research was supported by National Institute of Mental Health Grant MH-15142 to the second author. for specific memory. As an alternative, Reitman and Bower propose an explanation based upon simple frequency considerations. According to their tally model, subjects during acquisition attach familiarity tags to each sentence as a whole, and such tags generalize to ordered subunits of the sentences as well, down to single-idea units. At testing, subjects first decompose the test sentence into single-idea units. Each single element is checked for the frequency of associated familiarity tags and is recognized as old if the frequency exceeds a certain criterion; otherwise, it is considered new. If any single-idea unit is not recognized, the sentence is called new. If all are recognized, the analysis proceeds to the next higher order subunit, that is, digrams. Each digram is assessed for familiarity tag frequency, and a running tally begins. The tally is incremented by one whenever a given digram has a familiarity above a certain threshold; otherwise, it is decremented by one. The process is repeated for trigrams, and so on, until the sentence itself is assessed. Thus, for a sentence composed of ideas A, B, and C, in that order, after checking each single idea for familiarity tags, $A B$ and $B C$ subunits would be checked, and, finally, the entire sentence, ABC, would be evaluated. A positive recognition response is given only if the total tally exceeds a certain criterion.

As yet, there has been no adequate test of these two theories in a situation where they predict contrary results. Flagg (1976) attempted such a test, but his study contained serious confoundings. The purpose of this study was to provide such a test.

In this experiment, use was made of the fact that, in the Reitman and Bower (1973) theory, both formation of familiarity tags and analyses of test sentences occur in terms of the ordered subunits. Thus, the basic Bransford and Franks (1971) paradigm was used, but 
with two groups varying in the nature of the acquisition sentences encountered. For one group (adjacent), all acquisition sentences were composed only of ideas that were contiguous in the original, four-idea reference sentences. For the other, nonadjacent group, all acquisition sentences were composed of ideas that were noncontiguous in the four-idea sentences. According to Bransford and Franks, integration should occur for both groups, and therefore, the usual results, that is, recognition ratings linearly increasing with number of ideas and no old-new differences for test sentences, should eventuate for both. The tally model, though, predicts that both groups will show a negative relation between recognition ratings and number of ideas for new sentences up to three ideas in complexity. This is due to the fact that, for the adjacent group, new test sentences will be primarily composed of nonadjacent subunits. Thus, not having been present during acquisition, these subunits will produce decrements in the overall tally, with more subunits leading to a greater decrement. The same is true of the nonadjacent group, for whom new test sentences will contain mainly adjacent subunits. For both groups, the slope of the line relating confidence rating and complexity for new sentences should be negative for complexities up to three ideas in length. For four-idea sentences, the two groups should show differing behavior. The adjacent group should give higher confidence ratings to these than to any other sentences, since most of the subunits will have been seen in acquisition (see Table 1). In contrast, the nonadjacent group should give lower confidence ratings to these sentences than to any other, because the majority of the subunits will never have been seen (Table 1). The following study was designed to evaluate the relative merit of the two theories.

\section{METHOD}

\section{Subjects}

Subjects were 14 undergraduate volunteers from introductory psychology classes at the University of New Mexico. Of these, seven were assigned to each of the adjacency groups.

\footnotetext{
Materials

Acquisition and recognition sentences were all derived from four different reference sentences, each of which contained four simple ideas. The set of acquisition sentences for each subject consisted of 24 different sentences, with two "ones" (one-idea sentences), two "twos" (two-idea sentences), and two "threes" (three-idea sentences) from each reference sentence. These were randomly ordered, with the constraint that no two
}

consecutive sentences contain ideas from the same reference sentence. There were two different types of acquisition sentences, adjacent and nonadjacent. The former consisted entirely of sentences the simple ideas of which were contiguous in the given reference sentence. The nonadjacent sentences contained sets of ideas that were noncontiguous in the given reference sentence. Table 1 provides an example of these two types of lists in schematic form. Thus, for example, the sentence, "The girl found a rubber ball between the trees in the park," can be represented as an ordered concatenation of the simple ideas $\mathrm{ABCD}$, where these ideas are, respectively, "The girl found a ball," "The ball was rubber," "The ball was between the trees," and "The trees were in the park." Also listed in Table 1 are the approximate tallies associated with each sentence when evaluated at recognition. The simplifying assumption is made that, if a given unit or subunit appeared either alone or as part of a larger idea set in acquisition, it adds one to the total tally; otherwise, one is subtracted. Other methods are, of course, possible, but do not change the ordinality of the predictions. As an example, test sentence $A B C D$ for the adjacent group would receive a tally of +4 , since all the subunits $A B, B C, C D$, $\mathrm{ABC}$, and $\mathrm{BCD}$ will have appeared during acquisition, while the whole set $A B C D$ will not, leaving $+5+-1=+4$.

The recognition test itself was composed of 54 sentences, with all 24 acquisition sentences, two new ones, two new twos, and two new threes from each reference sentence, the four reference sentences (fours), and six noncases, formed by combining simple ideas from each of two different reference sentences. Both acquisition and recognition sentences were recorded on tape, with 15 sec between each acquisition sentence and 5 sec between each recognition sentence.

\section{Procedure}

Subjects were run in groups of two to three. They were told they would hear a series of sentences for study, after each of which the word "now" would be spoken. After hearing this word, they were to write each sentence on the top sheet of a small tablet, then turn the page in preparation for the next sentence. After all 24 acquisition sentences had been presented over a centralized speaker, subjects were given a page containing a large number of simple multiplication problems on which they worked for $5 \mathrm{~min}$. They were then told that they were going to hear another set of sentences, all related to the sentences they had already heard, and were given an answer sheet with columns labeled "old" and "new" and rows of numbers from 1 to 5 down the right hand side. Their task was to put a check in the appropriate row of the "old" column if a given sentence was word for word the same as one presented in the first sentence set; if not, they were to put a check under "new." They also indicated their confidence in each response by circling a number from 1 to 5 , where 1 meant they were not at all sure, and 5 meant they were positive their answer was correct.

\section{RESULTS}

As is typically found, the proportion of noncases correctly identified as new was quite high: for the adjacent group, .81, and for the nonadjacent group,

Table 1

Acquisition and Test Sentences from Schematic Reference Sentence ABCD

\begin{tabular}{|c|c|c|c|c|c|c|c|c|}
\hline \multirow[b]{2}{*}{ Complexity } & \multicolumn{4}{|c|}{ Adjacent Group } & \multicolumn{4}{|c|}{ Nonadjacent Group } \\
\hline & Old Sentences & Tally & New Sentences & Tally & Old Sentences & Tally & New Sentences & Tally \\
\hline $\begin{array}{l}\text { One Idea } \\
\text { Two Ideas } \\
\text { Three Ideas } \\
\text { Four Ideas }\end{array}$ & $\begin{array}{l}A, B \\
A B, C D \\
A B C, B C D\end{array}$ & $\begin{array}{r}0 \\
+1 \\
+3\end{array}$ & $\begin{array}{l}\text { C, D } \\
\text { AC, BC } \\
\text { ABD, ACD } \\
\text { ABCD }\end{array}$ & $\begin{array}{r}0 \\
-1 / 2 \\
-1 \\
+4\end{array}$ & $\begin{array}{l}\mathrm{A}, \mathrm{C} \\
\mathrm{AC}, \mathrm{BD} \\
\mathrm{ABD}, \mathrm{ACD}\end{array}$ & $\begin{array}{r}0 \\
+1 \\
+3\end{array}$ & $\begin{array}{l}\text { B, D } \\
A B, B C \\
A B C, B C D \\
\text { ABCD }\end{array}$ & $\begin{array}{r}0 \\
-1 / 2 \\
-1 \\
-2\end{array}$ \\
\hline
\end{tabular}


1.00. Concerning the best dependent measure for sentence recognition, James, Hillinger, and Murphy (1977) have pointed out that proportion of "old" responses provides the most readily interpretable measure. Since both the Bransford and Franks (1971) theory and the tally model make their predictions in terms of confidence ratings, it was felt most appropriate to use weighted confidence ratings instead (Franks \& Bransford, 1974). These are obtained simply by multiplying confidence ratings for "new" responses by -1 and using the confidence ratings for "old" responses directly. Using this measure, an analysis of variance was conducted, with a between-subjects factor of adjacency and two within-subjects factors of old-new and complexity (ones through threes). At the $\mathrm{p}<.05$ level, the following effects were found to be significant. "Old" responses were given higher confidence ratings than "new" responses $[\mathrm{F}(1,12)=15.47, \mathrm{MSe}=2.54]$. More complex sentences were given higher ratings than less complex sentences $[F(2,24)=29.89, \mathrm{MSe}=1.92]$. There was also an interaction of old-new and adjacency, owing to the fact that the old-new difference was far more pronounced for the adjacent than for the nonadjacent group $[\mathrm{F}(1,12)=4.93, \mathrm{MSe}=2.54]$. The effect of adjacency was only marginally significant $[\mathrm{F}(1,12)=4.57, \mathrm{MSe}=5.23, \mathrm{p}<.054]$. These results are depicted in Figure 1.

An analysis of the significance of the linear trend component was also employed separately for "old" and "new" responses for each adjacency condition. For all four curves, this component was significant $(p<.01)$. For the adjacent group, for "old" and "new" responses, the respective tests showed $F(1,24)=8.81$ $(\mathrm{MSe}=2.70)$ and $\mathrm{F}(1,24)=14.10(\mathrm{MSe}=2.70)$. For the nonadjacent group, "old" responses yielded $\mathrm{F}(1,24)=18.55 \quad(\mathrm{MSe}=2.70)$ and new responses, $\mathrm{F}(1,24)=44.28(\mathrm{MSe}=2.70)$. For all four curves, the slope was positive, and for the "new" responses of both the adjacent and the nonadjacent groups, the four-idea

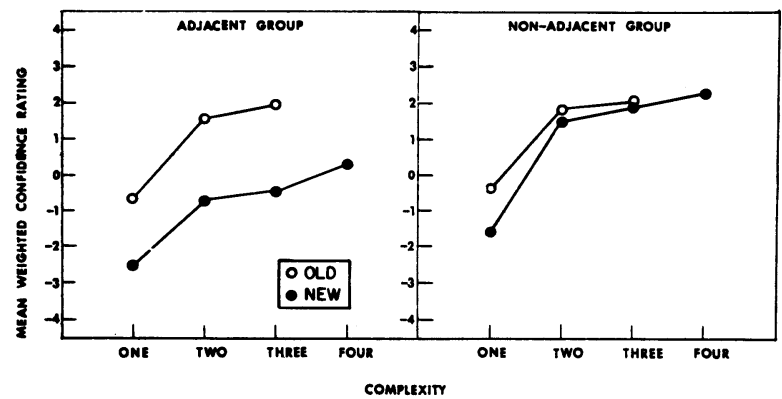

Figure 1. Mean weighted confidence ratings for adjacent and nonadjacent groups. reference sentences were given the highest confidence ratings.

\section{DISCUSSION}

There are two noteworthy facts apparent in the results. First, for both the adjacent and the nonadjacent groups, recognition ratings were found to increase with increasing sentence complexity. Second, for the adjacent group, "old" responses were given consistently higher confidence ratings than were "new" responses, whereas for the nonadjacent group, there was essentially no difference. These findings are more consistent with the Bransford and Franks (1971) position.

Both major predictions of the Reitman and Bower (1973) model were disconfirmed. For new sentences, the slope for recognition ratings was positive rather than negative for both groups. Also, although for the adjacent group, the confidence rating was higher for the fours than for any other level of complexity among new sentences, consistent with the tally model, this was also true of the nonadjacent group, for which the tally model predicted the lowest confidence rating. The tally model seems quite inadequate, then, as evaluated in this paradigm.

The theory of Bransford and Franks (1971) does not escape unscathed, however, since the second finding was not anticipated by either model. That is, it is not clear why there should be an old-new separation for the adjacent group and not for the nonadjacent group. Within the Bransford and Franks view, there are two not necessarily independent explanations of this outcome. One possibility is that sentences that contain noncontiguous ideas play an important causal role in the integration of related ideas into a single schema. It is plausible that, given a series of such sentences from a single reference sentence, there is in some sense a greater amount of overlap between these sentences than would be present for a series of sentences containing contiguous ideas, and this, in turn, might lead to more complete integration of those ideas into one conceptual unit. Another, related explanation is simply that memory for verbatim form is more likely to be retained when acquisition sentences contain only contiguous ideas from the reference sentences

\section{REFERENCES}

Bransford, J. D., \& Franks, J. J. The abstraction of linguistic ideas. Cognitive Psychology, 1971, 2, 331-350.

FLAGG, P. W. Semantic integration in sentence memory? Journal of Verbal Learning and Verbal Behavior, 1976, 15, 491-504.

Franks, J. J., \& Bransford, J. D. A brief note on linguistic integration. Journal of Verbal Learning and Verbal Behavior, $1974,13,217-219$.

James, C. T., Hillinger, M. L., \& Murphy, B. J. The effects of complexity on confidence ratings in linguistic integration. Memory \& Cognition, 1977, 5, 355-361.

ReItman, J., \& Bower, G. H. Storage and later recognition of exemplars of concepts. Cognitive Psychology, 1973, 4, 194-206.

(Received for publication August 7, 1978.) 